\title{
Double Phosphaalkyne Insertion to a Tungsten Alkylidyne: Formation of a Diphosphametallabicyclobutane
}

\author{
G. M. Jamison* and D. R. Wheeler \\ Sandia National Laboratories, Chemical Synthesis and Nanomaterials, Organization \\ 1846 \\ MS 0888, P. O. Box 5800 \\ Albuquerque, New Mexico 87185-0888 \\ Douglas A. Loy* \\ Los Alamos National Laboratory, Polymers and Coatings Group \\ MS E549 Los Alamos, New Mexico 87154 \\ Joseph W. Ziller \\ Department of Chemistry \\ University of California, Irvine \\ Irvine, California 92697-2025
}

\section{CONTENTS}

Synthesis, Spectral and Analytical Data for 3C.

Synthesis, Spectral and Analytical Data for $\mathbf{4 C}$.

Structure Determination Summary for $\mathbf{4 C}$.

Data Collection and Reduction Summary for $\mathbf{4 C}$.

Solution and Refinement Summary for $\mathbf{4 C}$.

Table 1. Experimental Data for the X-ray Diffraction Study of $\mathbf{4 C}$.

Table 2. Complete Experimental Data for the X-ray Diffraction Study of $4 \mathrm{C}$. 
Table 3.

Table 4.

Table 5.

Table 6.
Complete Atomic Positional Parameters for 4C.

Complete Interatomic Distances $(\AA)$ and Angles for 4C.

Complete Anisotropic Displacement Coefficients for $\mathbf{4 C}$.

H-Atom Coordinates and Isotropic Displacement Coefficients for $4 \mathrm{C}$. 
$[\mathrm{ArN}]\left[\left(\mathrm{F}_{3} \mathrm{C}\right)_{2} \mathrm{MeCO}\right] \mathrm{W}=\mathrm{C}(\mathrm{t}-\mathrm{Bu})-\mathrm{P}\left(\mathrm{OCMe}\left(\mathrm{CF}_{3}\right)_{2}\right)-\mathrm{C}(\mathrm{H})(\mathrm{t}-\mathrm{Bu}) \mathbf{3 C}$ and $[\mathrm{ArN}]\left[\left(\mathrm{F}_{3} \mathrm{C}\right)_{2} \mathrm{MeCO}\right]_{2} \mathrm{~W}\{-\mathrm{C}(\mathrm{t}-\mathrm{Bu})-\mathrm{P}(-\mathrm{C}(\mathrm{t}-\mathrm{Bu})=\mathrm{C}(\mathrm{H})(\mathrm{t}-\mathrm{Bu}))-\mathrm{P}-\}$ 4C. Under an argon atmosphere (glove box), a $25 \mathrm{~mL}$ scintillation vial was charged with a magnetic stir bar, $0.108 \mathrm{~g}(0.136 \mathrm{mmol})$ of $[\mathrm{ArN}]\left[\left(\mathrm{F}_{3} \mathrm{C}\right)_{2} \mathrm{MeCO}\right]_{2} \mathrm{~W}=\mathrm{C}(\mathrm{H})(\mathrm{t}-\mathrm{Bu})(\mathbf{C})$, and $1.0 \mathrm{~mL}$ of dry benzene. At $25{ }^{\circ} \mathrm{C}, 4.1 \mu \mathrm{L}(0.408 \mathrm{mmol})$ of t-butylphosphaacetylene (1) was added and the vial was capped; the catalyst solution changed from bright yellow to dark yellow. After stirring $2 \mathrm{~h}$, solvent removal under vacuum left a orange residue which was dissolved in a minimum of dry pentane. Two recrystallizations by cooling at $-40{ }^{\circ} \mathrm{C}$ yielded $0.022 \mathrm{~g}$ of $\mathbf{4 C}$ as red crystals (16\% yield based on starting tungten alkylidene $\mathbf{C}$ ) and yellow crystals of $\mathbf{3 C}$ recovered from the pentane liquor.

Spectroscopic data for 3C: ${ }^{1} \mathrm{H}$ NMR (300 MHz, C6D6): $\delta=7.05$ (m, 3H, [(2,6-(iPr) $\left.\left.\left.{ }_{2} \mathrm{C}_{6} H_{3}\right) \mathrm{N}\right] \mathrm{W}\right), 4.35\left(\mathrm{~d}, 1 \mathrm{H},{ }^{2} \mathrm{JP}-\mathrm{H}=7 \mathrm{~Hz},{ }^{2} \mathrm{JW}-\mathrm{H}=13 \mathrm{~Hz}, \mathrm{~W}-\mathrm{C}(H)\left(\mathrm{C}\left(\mathrm{CH}_{3}\right) 3\right), 3.84\right.$ (septet, $\left.2 \mathrm{H},{ }^{3} \mathrm{~J}_{\mathrm{H}-\mathrm{H}}=7 \mathrm{~Hz},\left[\left(2,6-(i-P r)_{2} \mathrm{C}_{6} \mathrm{H}_{3}\right) \mathrm{N}\right] \mathrm{W}\right), 1.73\left(\mathrm{~s}, 3 \mathrm{H}, \mathrm{P}\left(\mathrm{OCCH}_{3}\left(\mathrm{CF}_{3}\right) 2\right)\right.$ ), $1.43\left(\mathrm{~s}, 3 \mathrm{H},\left[\left(\mathrm{F}_{3} \mathrm{C}\right)_{2} \mathrm{CH}_{3} \mathrm{CO}\right] \mathrm{W}\right), 1.39\left(\mathrm{~s}, 9 \mathrm{H}, \mathrm{W}\left(=\mathrm{C}\left(\mathrm{C}\left(\mathrm{CH}_{3}\right) 3\right)\right)\right), 1.35,1.19$ (each a d, $\left.12 \mathrm{H},{ }^{3} \mathrm{~J}_{\mathrm{H}-\mathrm{H}}=7 \mathrm{~Hz},\left[\left(2,6-(i-P r){ }_{2} \mathrm{C}_{6} \mathrm{H}_{3}\right) \mathrm{N}\right] \mathrm{W}\right), 0.90(\mathrm{~s}, 9 \mathrm{H}, \mathrm{W}-\mathrm{C}(\mathrm{H})(\mathrm{C}(\mathrm{CH} 3) 3)) .{ }^{13} \mathrm{C}$ NMR (75 MHz, C6 $\left.\mathrm{D}_{6}\right): \delta=234.5\left(\mathrm{~d},{ }^{1} \mathrm{JP}-\mathrm{C}=83 \mathrm{~Hz}, \mathrm{~W}\left(=C\left(\mathrm{C}\left(\mathrm{CH}_{3}\right) 3\right), 151.9,145.1\right.\right.$, $126.8,123.4\left(\left[\left(2,6-(\mathrm{i}-\mathrm{Pr})_{2} \mathrm{C}_{6} \mathrm{H}_{3}\right) \mathrm{N}\right] \mathrm{W}\right), 124.5,122.1\left(\right.$ each a q, ${ }^{1} \mathrm{~J}_{\mathrm{C}-\mathrm{F}}=284 \mathrm{~Hz}$, $\mathrm{P}\left(\mathrm{OCCH}_{3}\left(\mathrm{CF}_{3}\right) 2\right),\left[\left(\mathrm{F}_{3} \mathrm{C}_{2} \mathrm{CH}_{3} \mathrm{CO}\right] \mathrm{W}\right), 123.6\left(\mathrm{~d},{ }^{2} \mathrm{~J}_{\mathrm{C}-\mathrm{P}}=68 \mathrm{~Hz}, \mathrm{~W}-\mathrm{C}(\mathrm{H})\left(\mathrm{C}\left(\mathrm{CH}_{3}\right) 3\right)\right.$, 81.9 (septet, $\left.{ }^{2} \mathrm{~J} \mathrm{C}-\mathrm{F}=30 \mathrm{~Hz},\left[\left(\mathrm{~F}_{3} \mathrm{C}\right)_{2} \mathrm{CH}_{3} \mathrm{CO}\right] \mathrm{W}\right), 78.7$ (doublet of septets, ${ }^{2} \mathrm{~J} \mathrm{C}-\mathrm{F}=30$ $\mathrm{Hz},{ }^{2} \mathrm{JP}_{\mathrm{P}} \mathrm{C}=4 \mathrm{~Hz}, \mathrm{P}\left(\mathrm{OCCH}_{3}\left(\mathrm{CF}_{3}\right)_{2}\right), 44.7\left(\mathrm{~d},{ }^{2} \mathrm{~J}_{\mathrm{C}-\mathrm{P}}=18 \mathrm{~Hz}, \mathrm{~W}\left(=\mathrm{C}\left(C\left(\mathrm{CH}_{3}\right) 3\right)\right)\right), 38.6$ $\left(\mathrm{d},{ }^{2} \mathrm{~J}_{\mathrm{C}-\mathrm{P}}=12 \mathrm{~Hz}, \mathrm{~W}-\mathrm{C}(\mathrm{H})\left(\mathrm{C}\left(\mathrm{CH}_{3}\right) 3\right)\right), 34.6,33.9$ (each a doublet, ${ }^{3} \mathrm{~J}_{\mathrm{C}-\mathrm{P}}=5 \mathrm{~Hz}, 6 \mathrm{~Hz}$, $\mathrm{W}\left(=\mathrm{C}\left(\mathrm{C}\left(\mathrm{CH}_{3}\right) 3\right)\right)$ and $\left.\mathrm{W}-\mathrm{C}(\mathrm{H})\left(\mathrm{C}\left(\mathrm{CH}_{3}\right) 3\right)\right), 28.0,24.7,24.0$ (each a singlet, [(2,6- $(i-$ $\left.\left.\left.\operatorname{Pr})_{2} \mathrm{C}_{6} \mathrm{H}_{3}\right) \mathrm{N}\right] \mathrm{W}\right), 18.6\left(\mathrm{~s},\left[\left(\mathrm{~F}_{3} \mathrm{C}\right)_{2} \mathrm{CH}_{3} \mathrm{CO}\right] \mathrm{W}\right), 15.2\left(\mathrm{~d},{ }^{1} \mathrm{JP}-\mathrm{C}=16 \mathrm{~Hz}\right.$, $\left.\mathrm{P}\left(\mathrm{OCCH}_{3}\left(\mathrm{CF}_{3}\right)_{2}\right)\right)$. ${ }^{31} \mathrm{P} \mathrm{NMR}\left(121.4 \mathrm{MHz}, \mathrm{C}_{6} \mathrm{H}_{6}\right.$, ext. $\left.\mathrm{H}_{3} \mathrm{PO}_{4}\right): \delta=-106.4$ (s). Anal. Calcd for $\mathrm{C}_{30} \mathrm{H}_{42} \mathrm{NO}_{2} \mathrm{~F}_{12} \mathrm{PW}$ : C, 40.42; H, 4.75; N, 1.57. Found: C, 40.38; H, 4.71; N, 1.60 . 
Spectroscopic data for 4C: ${ }^{1} \mathrm{H}$ NMR $\left(300 \mathrm{MHz}, \mathrm{C}_{6} \mathrm{D}_{6}\right): \delta=6.96(\mathrm{~m}, 3 \mathrm{H},[(2,6-(\mathrm{i}-$ $\left.\left.\left.\operatorname{Pr})_{2} \mathrm{C}_{6} \mathrm{H}_{3}\right) \mathrm{N}\right] \mathrm{W}\right), 6.26\left(\mathrm{~d}, 1 \mathrm{H},{ }^{3} \mathrm{JP}-\mathrm{H}=33 \mathrm{~Hz}, \mathrm{~W}\{-\mathrm{C}(\mathrm{t}-\mathrm{Bu})-\mathrm{P}(-\mathrm{C}(\mathrm{t}-\mathrm{Bu})=\mathrm{C}(H)(\mathrm{t}-\mathrm{Bu}))-\mathrm{P}-\right.$ \}), 3.96 (septet, $\left.2 \mathrm{H},{ }^{3} \mathrm{~J}_{\mathrm{H}-\mathrm{H}}=7 \mathrm{~Hz},\left[\left(2,6-(i-\operatorname{Pr})_{2} \mathrm{C}_{6} \mathrm{H}_{3}\right) \mathrm{N}\right] \mathrm{W}\right), 1.80,1.76$ (each a s, $6 \mathrm{H}$, $\left.\left[\left(\mathrm{F}_{3} \mathrm{C}\right)_{2} \mathrm{CH}_{3} \mathrm{CO}\right]_{2} \mathrm{~W}\right), 1.40,1.39,1.28$ (each a s, $27 \mathrm{H}, \mathrm{W}\left\{-\mathrm{C}\left(\mathrm{CH}_{3}\right)-\mathrm{P}\left(-\mathrm{C}\left(\mathrm{CH}_{3}\right)=\mathrm{C}(\mathrm{H})\right.\right.$ $\left.\left.\left.\left(\mathrm{CH}_{3}\right)\right)-\mathrm{P}-\right\}\right), 1.29,1.26\left(\right.$ each a d, $\left.12 \mathrm{H},{ }^{3} \mathrm{~J}_{\mathrm{H}-\mathrm{H}}=7 \mathrm{~Hz},\left[\left(2,6-(i-P r){ }_{2} \mathrm{C}_{6} \mathrm{H}_{3}\right) \mathrm{N}\right] \mathrm{W}\right) .{ }^{13} \mathrm{C}$ NMR $\left(75 \mathrm{MHz}, \mathrm{C}_{6} \mathrm{D}_{6}\right): \delta=154.1\left(\mathrm{~d},{ }^{2} \mathrm{~J}_{\mathrm{P}-\mathrm{C}}=20 \mathrm{~Hz}, \mathrm{~W}\{-\mathrm{C}(\mathrm{t}-\mathrm{Bu})-\mathrm{P}(-\mathrm{C}(\mathrm{t}-\mathrm{Bu})=C(\mathrm{H})(\mathrm{t}-\right.$ $\mathrm{Bu}))-\mathrm{P}-\}), 151.3,147.8,128.8,123.6\left(\left[\left(2,6-(\mathrm{i}-\mathrm{Pr})_{2} \mathrm{C}_{6} \mathrm{H}_{3}\right) \mathrm{N}\right] \mathrm{W}\right), 134.2\left(\mathrm{~d},{ }^{1} \mathrm{~J}_{\mathrm{P}-\mathrm{C}}=120 \mathrm{~Hz}\right.$, $\mathrm{W}\{-\mathrm{C}(\mathrm{t}-\mathrm{Bu})-\mathrm{P}(-C(\mathrm{t}-\mathrm{Bu})=\mathrm{C}(\mathrm{H})(\mathrm{t}-\mathrm{Bu}))-\mathrm{P}-\}), 123.9,123.6\left(\right.$ each a $\mathrm{q},{ }^{1}{ }_{\mathrm{J}} \mathrm{C}-\mathrm{F}=214 \mathrm{~Hz}$, $\left[\left(\mathrm{F}_{3} \mathrm{C}\right)_{2} \mathrm{CH}_{3} \mathrm{CO}\right]_{2} \mathrm{~W}$ ), 118.7 (doublet of doublets, ${ }^{1} \mathrm{JP}_{\mathrm{P}-\mathrm{C}}=89 \mathrm{~Hz},{ }^{1} \mathrm{JP}-\mathrm{C}=70 \mathrm{~Hz},{ }^{1} \mathrm{JW}-$ $\mathrm{C}=70 \mathrm{~Hz}, \mathrm{~W}\{-C(\mathrm{t}-\mathrm{Bu})-\mathrm{P}(-\mathrm{C}(\mathrm{t}-\mathrm{Bu})=\mathrm{C}(\mathrm{H})(\mathrm{t}-\mathrm{Bu}))-\mathrm{P}-\}), 82.8,82.5\left(\right.$ each a septet, ${ }^{2} \mathrm{~J} \mathrm{C}-\mathrm{F}=$ $\left.45 \mathrm{~Hz},\left[\left(\mathrm{~F}_{3} \mathrm{C}\right)_{2} \mathrm{CH}_{3} \mathrm{CO}\right]_{2} \mathrm{~W}\right), 41.8,40.6,34.3$ (each a s, $\mathrm{W}\left\{-\mathrm{C}\left(\mathrm{C}\left(\mathrm{CH}_{3}\right) 3\right)-\mathrm{P}(-\right.$ $\left.\left.\left.\mathrm{C}\left(\mathrm{C}\left(\mathrm{CH}_{3}\right) 3\right)=\mathrm{C}(\mathrm{H})\left(C\left(\mathrm{CH}_{3}\right) 3\right)\right)-\mathrm{P}-\right\}\right), 34.7,32.7,31.9$ (each a s, $\mathrm{W}\left\{-\mathrm{C}\left(\mathrm{C}\left(\mathrm{CH}_{3}\right) 3\right)-\mathrm{P}(-\right.$ $\left.\left.\left.\mathrm{C}\left(\mathrm{C}\left(\mathrm{CH}_{3}\right) 3\right)=\mathrm{C}(\mathrm{H})\left(\mathrm{C}\left(\mathrm{CH}_{3}\right) 3\right)\right)-\mathrm{P}-\right\}\right), 32.6,28.2,25.6,24.0$ (each a s, [(2,6- $(i-$ Pr $\left.\left.\left.)_{2} \mathrm{C}_{6} \mathrm{H}_{3}\right) \mathrm{N}\right] \mathrm{W}\right), 18.1,17.5$ (each a s, $\left.\left[\left(\mathrm{F}_{3} \mathrm{C}\right)_{2} \mathrm{CH}_{3} \mathrm{CO}\right]_{2} \mathrm{~W}\right) .{ }^{31} \mathrm{P}$ NMR $(162.0 \mathrm{MHz}$, $\mathrm{CDCl}_{3}$, ext. $\left.\mathrm{H}_{3} \mathrm{PO}_{4}\right): \delta=-174.2$ (doublet, ${ }^{1} \mathrm{~J}_{\mathrm{P} \cdot \mathrm{P}}=162 \mathrm{~Hz}, \mathrm{~W}\{-\mathrm{C}(\mathrm{t}-\mathrm{Bu})-\mathrm{P}(-\mathrm{C}(\mathrm{t}-$ $\mathrm{Bu})=\mathrm{C}(\mathrm{H})(\mathrm{t}-\mathrm{Bu}))-P-\}),-184.8$ (doublet of quartets, ${ }^{1} \mathrm{~J}_{\mathrm{P}-\mathrm{P}}=162 \mathrm{~Hz},{ }^{6} \mathrm{~J}_{\mathrm{P}-\mathrm{F}}=16 \mathrm{~Hz}, \mathrm{~W}\{-\mathrm{C}(\mathrm{t}-$ $\mathrm{Bu})-P(-\mathrm{C}(\mathrm{t}-\mathrm{Bu})=\mathrm{C}(\mathrm{H})(\mathrm{t}-\mathrm{Bu}))-\mathrm{P}-\})$. Anal. Calcd for $\mathrm{C}_{35} \mathrm{H}_{51} \mathrm{NO}_{2} \mathrm{~F}_{12} \mathrm{P}_{2} \mathrm{~W}: \mathrm{C}, 42.40 ; \mathrm{H}$, 5.18; N, 1.41. Found: C, 42.61; H, 5.22; N, 1.37 . 